\title{
CUIDADO AOS ADOLESCENTES NA ATENÇÃO PRIMÁRIA: PERSPECTIVAS DE INTEGRALIDADE
}

\author{
Care to adolescents in primary care: comprehensive perspectives \\ Cuidado a los adolescentes en la atención primaria: perspectivas de la integralidad
}

\section{RESUMO}

0 estudo tem como objetivo descrever ações dos gestores e enfermeiros com os adolescentes na atenção primária, baseadas nas perspectivas da integralidade. Pesquisa qualitativa com quatro gestores e treze enfermeiros da rede básica de saúde realizada no período de abril a junho de 2010, utilizando entrevista e análise de conteúdo. Da análise emergiu a temática: Ações de cuidados ao adolescente e perspectivas de integralidade. Os sujeitos destacaram o acolhimento como uma ação da equipe, porém com necessidade de estabelecer vínculos e oportunizar meios para motivar a reflexão dos adolescentes acerca de sua responsabilidade mediante as questões de saúde. Tais ações conduzem a possibilidade da integralidade do cuidado. Concluiu-se que os profissionais mostraram as limitações do cuidado a esta população e apresentaram o confronto entre o pensar e o fazer, favorecendo reflexões sobre estratégias que possibilitem o cuidado aos adolescentes na perspectiva de integralidade.

Palavras-chave: Enfermagem. Adolescente. Assistência integral à saúde.

\begin{abstract}
The study has as objective to describe the actions of managers and nurses with adolescents in primary care, based on the comprehensive perspectives care. It's a qualitative research with four managers and thirteen nurses from the primary health care net, carried out from April to June 2010, using interview and content analysis. From the analysis the following theme emerged: Actions of care to adolescents and comprehensive perspectives care. The participants emphasized reception as a team action, but with the need to establish bonds and create tactics to motivate the reflection on adolescents about their responsibility on health issues. Such actions lead to the possibility of comprehensive care. We concluded that the professionals showed the limitations of care to this population and presented the comparison between thinking and acting, encouraging reflections on strategies that support the care of adolescents in the comprehensive perspective care.
\end{abstract}

Keywords: Nursing. Adolescent. Comprehensive Health Care.

\section{Resumen}

El estudio tiene como objetivo describir las acciones de los directivos y enfermeros con adolescentes en la atención primaria basadas en la discusión de las perspectivas de integridad. Investigación cualitativa con los cuatro directores y trece enfermeros de atención primaria de salud, realizada en el período de abril hasta junio de 2010, a través de entrevistas y análisis de contenido. Del análisis surgieron los temas: Las acciones de atención a los adolescentes y las perspectivas de integralidad. Los sujetos destacaron la acogida como acción del equipo, pero con necesidad de establecer vínculos y creación de oportunidades para motivar a la reflexión de los adolescentes acerca de su responsabilidad por los problemas de salud. Estas acciones conducen a la posibilidad de una atención integral. Se concluyó que los profesionales percibieron limitaciones de atención de esta población y presentaron la comparación entre el pensar y hacer, para promover la reflexión sobre las estrategias que faciliten el cuidado de los adolescentes en la perspectiva de la integralidad.

Palabras clave: Enfermería. Adolescente. Atención Integral de Salud.

\footnotetext{
${ }^{1}$ Enfermeira. Mestre em Cuidados Clínicos em Saúde e Enfermagem, Universidade Estadual do Ceará (UECE). Doutoranda da Escola de Enfermagem Anna Nery. Rio de Janeiro - RJ. Brasil. E-mail: rachelfranklincosta@hotmail.com; ²Enfermeira. Doutora em Enfermagem. Professora Adjunta do Curso de Graduação em Enfermagem e do Programa de Pós-Graduação Cuidados Clínicos em Enfermagem e Saúde do Centro de Ciências da Saúde da UECE. Fortaleza-CE. Brasil. E-mail: veraciog@hotmail.com; Doutora em Enfermagem. Professor Associado I, EEAN, Universidade Federal do Rio de Janeiro (UFRJ). Rio de Janeiro - RJ. Brasil. E-mail: regina.zeitoune@gmail.com
} 


\section{INTRODUÇÃO}

A integralidade é um princípio do Sistema Único de Saúde (SUS) e se configura como condição essencial para a assistência de adolescentes e jovens por orientar a organização dos serviços em seus diversos níveis de complexidade, assim como ampliar a visão sobre os determinantes biopsicossociais relacionados às necessidades de saúde desta faixa ${ }^{1}$.

A proteção integral da criança e do adolescente está postulada no Estatuto da Criança e do Adolescente (ECA), que os reconhece como sujeitos sociais, portadores de direitos e garantias próprias, independentes de seus pais e/ou familiares e do próprio Estado. Este estabeleceu obrigações diferenciadas para o Estado, as famílias e a sociedade em geral, configurando a denominada Doutrina da Proteção Integral. 0 ECA resultou de uma luta ampla dos setores sociais organizados que buscaram elaborar um novo espaço político e jurídico para a criança e 0 adolescente brasileiros e constitui uma legislação que visa ao seu desenvolvimento integral ${ }^{2}$.

A Organização Mundial de Saúde (OMS) define a adolescência como um processo fundamentalmente biológico, no qual se aceleram o desenvolvimento cognitivo e a estruturação da personalidade 3 . Esta faixa etária passa por modificações físicas, psicológicas e sociais, possui propensão a formar grupos, surgimento de desejos, descoberta de afinidades e reflexões sobre planos para a vida ${ }^{4}$.

Percebe-se que ainda existem lacunas nas práticas de cuidados destinadas aos adolescentes, de forma a não atenderem peculiaridades deste intervalo de idade. Não há um atendimento de forma sistematizada, e sim de acordo com a demanda da unidade, pois existem prioridades a outros grupos populacionais, razão por que a organização de trabalho com esse grupo etário fica a desejar. Nesse sentido, ao ampliar o campo de atenção e cuidado, poder-se-á contemplar o adolescente na agenda do serviço de saúde, favorecendo o aumento da procura e, consequentemente, o crescimento da oferta de serviços nas unidades básicas de saúde e respectivas áreas de abrangência 5 .

Nesta direção, os profissionais devem atuar de forma a inserir em sua prática uma abordagem integral, o que envolve a amplitude de visão acerca do conceito de saúde, desenvolvimento de trabalho em equipe e modificações na produção do cuidado, regida pela focalização das necessidades dos pacientes em suas diversas dimensões ${ }^{6}$. Assim, a equipe de saúde deve planejar e executar um cuidado baseado na integralidade, favorecendo a identificação e o estabelecimento de redes de apoio que facilitem e propiciem resultados que podem propiciar resultados mais efetivos? ${ }^{7}$.

As ações embasadas pelas noções da integralidade da atenção proporcionam a reorientação do planejamento de saúde para uma base populacional específica, como o adolescente, que poderá ensejar a promoção da saúde com medidas gerais e a proteção com medidas específicas para a prevenção de agravos e para a realização do cuidado clínico.

Ampliando essas concepções, o cuidado da enfermagem deve ser ético, pressupondo habilidades técnicas, conhecimento, sensibilidade e experiências, e guiado por ações que assistam, ofereçam supor te e facilitem ou melhorem a vida de quem é atendido em suas necessidades. Engloba a saúde em sua integralidade, nos seus aspectos psicobiológicos, espirituais, sociais e políticos ${ }^{9}$.

Dessa forma, os profissionais atuantes na atenção primária devem buscar conhecimentos e constituir uma prática baseada nos dispositivos legais da atenção integral que demandam uma qualificação técnica e científica na identificação dos riscos, percepção sobre os determinantes sociais de saúde e identificação da dinâmica familiar como orientadora do cuidado e de trabalho em equipe ${ }^{10}$.

Efetivamente, o ser que recebe o cuidado está inserido no sistema de saúde, portanto caminha por linhas de cuidado em diferentes níveis de atenção, que nem sempre são acessíveis. Isso porque os diferentes profissionais e serviços envolvidos no processo do cuidado integral estão muitas vezes interligados por burocráticas relações, não responsivas e não acolhedoras, caracterizadas por encaminhamentos e informações desencontradas, implicando a falta de responsabilização no atendimento dos usuários ${ }^{11}$.

Considerando as dificuldades profissionais em cuidar do adolescente, conforme observado na prática, considera-se necessário que tal problemática seja discutida no planejamento institucional e compartilhada entre os gestores e os enfermeiros, possibilitando o enfrentamento e possíveis soluções.

Ante tais considerações, percebeu-se a necessidade de conhecer as ações do enfermeiro e sua relação com a gestão do cuidado ao adolescente na atenção primária, desde 0 questionamento: Como se dá o processo de cuidar do enfermeiro e do gestor ao adolescente na atenção primária?

Ao iniciar a formulação do objeto deste estudo, foi realizado um levantamento nos bancos de dados na área de Enfermagem e nas principais bases de dados do campo da saúde, com recorte temporal de 1990 a 2011. Após consulta às terminologias em saúde na base de descritores da Biblioteca Virtual em Saúde (BVS), US National Library of Medicine (PUBMED), Cumulative Index to Nursing and Allied Health Literature (CINAHL), SCOPUS e Bireme (Decs), observou-se que os trabalhos encontrados não abordam as dimensões do cuidar integral ao adolescente na atenção primária. Portanto, viu-se a necessidade de se delinear um estudo voltado para esta temática, visto que implicará discussões e reflexões que possam subsidiar a melhoria da atenção a estes usuários.

Certamente, os achados e as reflexões emergentes ampliarão o conhecimento da realidade estudada, podendo contribuir com a melhoria do cuidado a essa população. No recorte da pesquisa ${ }^{a}$ para elaboração deste ar tigo, seguiu-se 
o objetivo: descrever ações dos gestores e dos enfermeiros com os adolescentes na atenção primária, baseadas nas perspectivas da integralidade.

\section{METODOLOGIA}

0 estudo foi descritivo na abordagem qualitativa, pois traz experiências dos sujeitos em um contexto social. Foi realizado em quatro Unidades Básicas de Saúde da Secretaria Executiva Regional IV (SER IV), conforme divisão da Secretaria de Saúde do Município de Fortaleza (Ceará), campo de prática do Curso de Graduação em Enfermagem da Universidade Estadual do Ceará (UECE). EstaSER possui 12 Unidades Básicas e um total de 42 enfermeiros atuando na Estratégia Saúde da Família (ESF).

Os participantes da pesquisa foram quatro gestores e 13 enfermeiros da rede básica de saúde que estavam diretamente envolvidos na organização dos serviços e no cuidado ao adolescente. A escolha destes profissionais foi intencional, atendendo aos critérios de tempo de atividade na atenção básica maior de seis meses e à disponibilidade destes em participar da pesquisa. Considerou-se este quantitativo conveniente, pois possibilitou a profundidade das informações obtidas. Estas foram coletadas por meio de entrevista semiestruturada, seguindo roteiro com as seguintes questões: Como se dá o cuidado que você desenvolve junto ao adolescente no plano assistencial? e Como se dá o cuidado que você desenvolve junto ao adolescente no plano gerencial?

A pesquisa foi realizada no período de abril a junho de 2010. As informações foram organizadas e interpretadas a partir do método de análise de conteúdo do tipo análise categorial temática, seguindo as fases de: pré-análise, exploração do material e tratamento dos resultados, análise e interpretação $0^{12}$. Na pré-análise foi realizada a organização do material a ser analisado, constituindo o corpus com os discursos dos sujeitos. Nesta etapa, foram realizadas leituras flutuantes do material empírico com o objetivo de preparar o material para a segunda etapa (leitura cuidadosa e construção das unidades de sentido). Nesta fase realizou-se a exploração do material com uma leitura exaustiva dos discursos e, em seguida, foram identificadas unidades de sentido, a fim de construir o inventário. Após esta etapa, realizou-se a codificação dos temas.

0 estudo foi oriundo do Projeto Saúde do Adolescente na Atenção Básica: linha do cuidado e sua interface com a rede assistencial, o qual foi aprovado pelo Comitê de Ética da Universidade Estadual do Ceará, sob protocolo número 09230911-9. Foi garantido anonimato dos participantes do estudo, respeitando a privacidade e a liberdade de participação ${ }^{13}$. Para preservar o anonimato, foi atribuída a cada um dos participantes identificação numérica, de acordo com a ordem de coleta de dados, aos gestores a letra $\mathrm{G}$ e aos enfermeiros a letra $\mathrm{E}$.

\section{RESULTADOS}

Os elementos da integralidade presentes nas ações dos enfermeiros e dos gestores nas práticas de cuidado ao adolescente e sua interface com a gestão estão descritos na temática: Ações de cuidados ao adolescente e perspectivas de integralidade. Nos discursos dos participantes, destacase 0 acolhimento realizado pelos profissionais, como uma etapa fundamental do cuidado.

Todos os pacientes que chegam aqui sejam os adolescentes, os adultos (...) ele vai para sua equipe. Vai para o enfermeiro que faz o acolhimento, e, se a pessoa está com alguma necessidade de cuidado maior, ele passa para o médico (G1).

Cada profissional faz a sua parte no acolhimento, tentamos conversar da melhor forma possível, sabendo que essa é uma etapa mais difícil da vida. A gente tenta tratar de forma mais carinhosa, mais acolhedora mesmo [...] (E3).

Expressou-se uma aproximação entre o enfermeiro e as ações de acolhimento. No entanto, esta foi evidenciada como atividade individual não institucionalizada e não direcionada a esta faixa etária, conforme se verificou nos discursos.

Não existe um acolhimento específico, eles são recebidos da mesma forma que os demais não só pela falta de espaço, mas não tínhamos os profissionais para receber dentro dessa questão (G4).

[...] não é um acolhimento específico. 0 acolhimento é sempre a enfermeira quem faz [...] (E8).

Alguns profissionais sinalizaram a inexistência de ações promotoras de acolhimento para os adolescentes, embora ressaltassem como ação fundamental.

Eles chegamna recepção, marcama consulta, enão existe acolhimento. Agente sabearealidade de cada um, a gente sabe muita coisa deles e trabalha individualmente. Não existem ações coletivas e nem voltadas para um grupo espećíico de adolescentes (E10).

Não temos acolhimento, e isso é muito importante numa unidade. Considero como uma forma de organizar o atendimento, de ver de quem é a prioridade, quem tem necessidade [...] (E12).

Mesmo diante de limitações, os profissionais mostraram a necessidade de estabelecer estratégias para formação de vínculo com os adolescentes. 
[...] o adolescente que a gente precisa ter mais contato, a gente anota o endereço para o agente de saúde ir lá e ver como ele está após a consulta. Sempre a gente tem essa preocupação de fazer 0 retorno [...]. A gente tem o compromisso e vínculo também com o familiar [...] (G3).

Em relação ao vínculo, ocorre com aqueles que são da área da unidade, principalmente os que são da equipe de saúde da família que tem uma ligação maior dos ACS com esses usuários (E13).

Por outro lado, há o reconhecimento dos profissionais acerca da inexistência do vínculo entre instituição/profissionais e adolescentes, apontando situações diferenciadas.

[...] fica difícil dizer quem é quem, mas não deixaram de ser atendidos dentro de um contexto geral [...] (G4).

Vínculo é muito difícil para mim porque desde que eu entrei já mudei de área de abrangência várias vezes [...]. Nós temos que criar vínculo com o adolescente, pois não é um cliente fácil (E12).

Os entrevistados em seus discursos alegaram que os agentes de saúde se responsabilizavam pela busca ativa por adolescentes.

0 acesso se dá de duas formas, ou por demanda livre ou trazidos pelos agentes de saúde, se for alguma coisa de emergência [...] ver como podemos resolver a questão (G4).

0 acesso é igual aos outros usuários. Chegam por demanda espontânea algumas vezes os agentes de saúde trazem e, também, quando são encaminhados pelas escolas próximas (E13).

Os profissionais retrataram a resolutividade como ferramenta para a integralidade, enfocando a referência e contrarreferência como situação real da prática.

[...] a resolutividade dos problemas é algo bem complexo. Adolescente com alguma característica de risco, a gente tenta fazer as referências [...]. A gente tenta abrir os campos de atenção à saúde que tem dentro da unidade para cada necessidade específica. [...] não tem contrarreferência, mas a gente está pedindo ao agente de saúde para fazer essa busca ativa do retorno do adolescente à unidade (E2).
Tudo que eu posso fazer e minha equipe pode fazer, se tiver como pedir a outros profissionais para resolver eu tento agilizar [...]. Eu tento entrar em contato com a médica, mas se tiver coisas mais importantes, a gente manda para a coordenação e aífica entre a coordenação e as parcerias (E5).

Os profissionais destacaram a importância de opor tunizar a ida do adolescente ao serviço para orientá-lo, constituindo um processo de corresponsabilização.

A gente trabalha a responsabilização sempre no contexto de corresponsabilidade [...] a gente tenta colocar para ele que a responsabilidade também é dele, que nós, enquanto profissionais, estamos aqui disponiveis para dar um atendimento da melhor forma possível [...] a gente vai tentando fortalecer essa questão da resolutividade utilizando essas nossas parcerias (E1).

Quanto à responsabilização, fica tanto comigo quanto com ele, como com o agente de saúde da área dele [...]. Temos que ficar vendo se foi resolvido, vendo se ele foi mesmo para onde foi encaminhado, tudo o que aconteceu, e eles (agentes) apresentam depois o resultado do que aconteceu com o adolescente (E5).

Os entrevistados reforçaram o desenvolvimento de ações que favoreciam a autonomia dos adolescentes em relação à sua própria saúde.

[...] estou sempre passando pra eles que vão ter que se cuidar, e se tiver com quem estar dividindo, tirando dúvidas, e que agora tem que ter realmente preocupação e cuidado com a saúde deles (E11).

Tento colocar para eles que estão gerindo a própria vida. A gente coloca as coisas que eles têm que assumir, pois não vamos estar ao lado deles 24 horas por dia. Quando existe um problema maior, a gente compartilha com a equipe para fazer os encaminhamentos, mas quando ele pode andar com as próprias pernas, a gente faz com que ele veja que ele é responsável pela vida dele (E13).

\section{DISCUSSÃO}

Entende-se a integralidade como um dos princípios que contribuiu consideravelmente para a reforma do sistema de saúde brasileiro, que envolve uma visão ampliada do cuidado e alguns atributos no que se refere às práticas profissionais e à organização do serviço. Portanto, a articulação dos conceitos 
de integralidade adotados na referida pesquisa foram acolhimento, vínculo/responsabilização e qualidade da atenção.

No sistema de saúde, o usuário é visto como aquele ser social que necessita de ações que promovam sua saúde. Nesta perspectiva, a Política Nacional de Atenção Básica (PNAB) inclui como fundamento a efetivação da integralidade em seus vários aspectos, como integrar ações programáticas e demanda espontânea; articular as ações de promoção da saúde, prevenção de agravos, vigilância à saúde, tratamento e reabilitação, trabalho de forma interdisciplinar e em equipe; $e$ coordenar o cuidado na rede de serviços ${ }^{14}$.

Neste sentido, os profissionais da atenção básica expuseram suas experiências quanto ao cuidado ao adolescente, as lacunas e as dificuldades no serviço para a efetivação da integralidade operacionalizada em diversas ações. Em relação ao acolhimento dos adolescentes, não existe um projeto institucional que oriente estas aç̃es, embora o enfermeiro seja um dos profissionais que aborda em seu processo de cuidar esta dimensão relacional.

A integralidade caracteriza-se pela apreensão das necessidades do sujeito a partir do encontro com a equipe de saúde; para tanto, são necessários novos modos de cuidados e de cuidar associando-se aos saberes técnicos, operantes, e às tecnologias relacionais e subjetivas ${ }^{15}$. Nesta perspectiva, 0 profissional aproxima-se das necessidades dos usuários, promovendo acolhimento e favorecendo o vínculo.

0 acesso do adolescente à atenção básica deve ser garantido, como também deve estar adequado às suas necessidades, contemplando a política de atenção à saúde desses jovens. 0 serviço deve promover ações que facilitem 0 engajamento do jovem em seu cuidado nas dimensões preventivas e de promoção da saúde. A partir do acesso e do acolhimento, os adolescentes constroem suas relações de vínculo com os profissionais de saúde e, desse modo, alcançam a autonomia para compartilhar a tomada de decisão sobre as possibilidades de preservar a saúde.

Apesar de o termo acolhimento denotar recebimento na entrada, na chegada ao serviço, é importante apontar para a necessidade de 0 acolhimento estar presente em todos os momentos do cuidado produzido na assistência à saúde do adolescente.

Entretanto, o processo de cuidado ainda se encontra estratificado, pois, muitas vezes, visualiza o sujeito como ser acometido por algum problema de saúde ou uma doença; e olvidam-se da subjetividade, esquecem-se de que ele tem uma história de vida, que é também determinante do seu processo de saúde e doença ${ }^{11}$.

Para que a integralidade seja realizada na prática profissional, é necessário que estes profissionais atuem de maneira interdisciplinar, atendendo às necessidades dos usuários, promovendo assistência qualificada que apresenta várias dimensões, mas inclui, essencialmente, acolhimento, vínculo e acesso.
Portanto, é importante que o adolescente se sinta aceito, confortado, ajudado e respeitado nos seus direitos para que possa confiar nos profissionais e retornar aos serviços de saúde. A forma como é recebido na unidade e acolhido por todos os profissionais de saúde irá definir o vínculo que será estabelecido com os profissionais e com a unidade.

Os profissionais reconheceram a significância do estabelecimento de vínculo e da necessidade de estratégias que impulsionam a presença dos adolescentes nos serviços de saúde, embora não o fizessem de forma específica para esta faixa etária, tratando-os como os usuários em geral. No entanto, os adolescentes necessitam de estímulos para se vincularem com os profissionais e os serviços como promoção de confiança e de atividades que 0 atraiam.

Para o alcance da integralidade, são necessários meios para a resolutividade e uma responsabilização por parte dos usuários, dos profissionais de saúde e do Estado. Acreditase que a inadequada capacitação dos profissionais envolvidos na operacionalização da ESF contribui para a pouca resolutividade das necessidades de saúde dos usuários. Assim, requer, por parte dos profissionais atuantes no sistema, conhecimentos das dimensões teóricas e práticas no desempenho de suas funções. Ante a insuficiente resolutividade do ESF, a população continua buscando por serviços de urgência e emergência para solucionar os problemas de saúde ${ }^{16}$.

Gestores e enfermeiros mencionaram que os agentes de saúde se responsabilizavam pela busca ativa por adolescentes. Portanto, demonstraram pouca resolutividade nas ações direcionadas ao cuidado integral deste grupo populacional. Para evitar esta pouca resolutividade, o profissional tem que estar atento e flexível para desenvolver suas atividades, conforme preconizam as políticas de saúde que estabelecem diretrizes e princípios capazes de promover a cidadania garantida na Constituição brasileira. Diante disso, aponta-se para a necessidade de os profissionais serem mais bem preparados e resolutivos, pois a abordagem aos adolescentes certamente requer todas essas qualidades profissionais ${ }^{16}$.

Voltando-se para a realidade dos profissionais entrevistados, percebeu-se a atuação por meio de parcerias com outras unidades e outros profissionais que integram a equipe interdisciplinar na tentativa de minimizar os problemas de saúde dos adolescentes, melhorando a resolutividade das ações e aproximando cada vez mais estes sujeitos das unidades básicas de saúde. Sabe-se que promover esclarecimentos e orientações aos adolescentes utilizando abordagem participativa pode facilitar a formação de sua autonomia, tornando-os corresponsáveis pela sua saúde.

Um estudo recente sobre o cuidado ao adolescente na atenção primária mostrou que a quantida-de de adolescentes que buscavam por atendimento nas unidades básicas representava uma pequena parcela do total de usuários que usufruíam diariamente dos serviços oferecidos. Assim, sugerem 
os autores que este nível de assistência deve ter um plano de ação subsidiado pela prática de acolhimento e vínculo que os motivem a participar, além de equipe completa, insumos e equipamentos adequados para o atendimento desta população ${ }^{17}$.

As práticas de saúde devem ser pautadas na produção da responsabilização clínica e da intervenção resolutiva por parte dos profissionais. 0 trabalhador de saúde, nesta perspectiva, deve ser resolutivo e servir como um facilitador de todo este processo, como forma de atingir a integralidade da atenção e autonomia do adolescente.

Ante os resultados apresentados, consideram-se algumas limitações no estudo, como a necessidade de estender os locais da coleta de dados para outras unidades básicas, ampliando o escopo dos sujeitos e possíveis variações nos achados. Foram selecionadas, no entanto, quatro Unidades consideradas representativas em relação à cobertura de famílias da área adscrita, oferta e dinamismo dos serviços. Na coleta e análise, foi percebida a saturação teórica das informações. Alguns discursos foram breves, mas mostraram a realidade dos sujeitos. Assim, os resultados trazem informações que podem subsidiar outras investigações no âmbito desta temática, ampliando o conhecimento sobre esta realidade, que pode ser explorada com outras nuanças.

\section{CONSIDERAÇÕES FINAIS}

0 objetivo da pesquisa foi contemplado nos discursos dos gestores e enfermeiros, quando expressaram as ações com os adolescentes na atenção primária, baseadas nos dispositivos da integralidade, como acolhimento, vínculo, acesso, entre outras ferramentas para a resolubilidade dos problemas de saúde deste grupo populacional.

A necessidade do trabalho em equipe com adoção de diretrizes do SUS, essencialmente, a possibilidade de integralidade, ausência de planejamento para suprir tais prerrogativas, incluindo ações definidas sobre o acolhimento e o vínculo, talvez sejam causas básicas que afastam os adolescentes, levando a pouca procura deste serviço.

Apesar de os entrevistados demonstrarem algumas ações de acolhimento, estas foram entendidas como atividades pontuais por meio de palestras, conversas, agendamentos de consultas realizados de forma individual, ou seja, distante de um planejamento institucional. Portanto, o acolhimento não esteve em conformidade com as diretrizes do princípio de integralidade, de modo a produzir a responsabilização dos profissionais com a saúde do adolescente.

0 vínculo e a autonomia foram apontados como ferramentas importantes na produção do cuidado ao adolescente, mas não se destacaram como ação efetiva e regular no fazer de todos os profissionais enfermeiros, nem foram assumidos pelos gestores.

Observou-se que os entrevistados se propuseram articular com outros setores e outros profissionais, promovendo o cuidado com ações ditas "integrais", com participação de todos os sujeitos envolvidos, proporcionando escuta e o cuidado individualizado. Esta prática em análise, entretanto, não foi efetivada conforme as necessidades dos adolescentes.

Os entrevistados apontaram a responsabilização pelo adolescente e, ao mesmo tempo, cer to envolvimento em seu cuidado, mas mostraram a necessidade de incluir mais os adolescentes nas situações de saúde, de modo a promover sua autonomia.

Observando que há pouca produção nesta temática, o estudo traz considerações sobre as ações dos gestores e dos enfermeiros com os adolescentes, evidenciando que há carências na organização dos serviços, de modo a contemplar o cuidado a esta população. Assim, os resultados indicaram os desafios a serem assumidos pelos enfermeiros e pelos gestores para fortalecer a atenção à saúde dos adolescentes nas Unidades Básicas fundamentadas no princípio da integralidade. Portanto, há necessidade de uma capacitação profissional envolvendo estes trabalhadores, de modo a ampliar ações e qualificá-los para o cuidado aos adolescentes. Isso possibilitaria a inserção dos adolescentes como sujeitos protagonistas do seu cuidado.

\section{REFERÊNCIAS}

1.Ministério da Saúde (BR). Saúde integral de adolescentes e jovens: orientações para a organização de serviços de saúde. Brasília(DF); 2005. (Série A - Normas e manuais técnicos)

2.Ministério da Saúde(BR). Lei Federal n8.069/90. Estatuto da criança e do adolescente. Brasilia(DF); 1990.

3.Taquette SR, Vilhena MM, Silva MM, Vale MP. Conflitos éticos no atendimento à saúde de adolescentes. Cad Saude Publica. 2005 nov; 21(6): 1717-25.

4.Horta N C, Madeira AMF, Armond LC. Desafios na atenção à saúde do adolescente In: Borges ALV, Fujimore E, organizadores. Enfermagem e a saúde do adolescente na atenção básica. São Paulo: Manole; 2009. p. 119-41.

5.Ferrari RAP, ThomsonZ, Melchior R. Atenção à saúde dos adolescentes: percepção dos médicos e enfermeiros das equipes da saúde da família. Cad Saude Publica. 2006 nov; 22(11): 2491-95.

6.Fontoura RT, Mayer CN. Uma breve reflexão sobre a integralidade. Rev Bras Enferm. 2006 jul; 59(4): 532-37.

7.Pedroso MLR, Motta MGC. Vulnerabilidades socioeconômicas e 0 cotidiano da assistência de enfermagem pediátrica: relato de enfermeiras. Esc. Anna Nery. 2010 jun; 14 (2): 293-300.

8.Ferrari RAP, Thomson Z, Melchior R. Adolescência: ações e percepção de médicos e enfermeiros do programa saúde da família. Interface: comunic saude educ. 2008 jun; 12(25): 387-400. 
9.Pinho IC, Siqueira JCBA, Pinho LMO. As percepções do enfermeiro acerca da integralidade da assistência. Rev Eletr Enferm. [on-line]. 2006; [citado 2011 mar 10]; 8(1): [aprox. 10 telas]. Disponível em: http:// www.revistas.ufg.br/index.php/fen/article/view/945/1156

10.Tavares MFL, Mendonça MHM, Rocha RM. Práticas em saúde no contexto de reorientação da atenção primária no Estado do Rio de Janeiro, Brasil, na visão das usuárias e dos profissionais de saúde. Cad Saude Publica. 2009 maio; 25(5): 1054-62.

11. Franco TB, Magalhães Júnior HM. Integralidade na assistência à saúde: a organização das linhas do cuidado. In: Merhy EE. et al. 0 trabalho em saúde: olhando e experienciando o SUS no cotidiano. São Paulo: Hucitec; 2007. p. 125-33.

12. Bardin L. Análise de conteúdo. Lisboa: Ed 70; 2009.

13. Ministério da Saúde (BR). Manual Operacional para Comitês de Ética em Pesquisa. Brasília(DF); 2002.

14. Ministério da Saúde(BR). Política Nacional da Atenção Básica. Brasilia(DF); 2006.

15. Sousa FGM, Erdmann AL, Mochel EG. Modelando a integralidade do cuidado à criança na Atenção Básica de Saúde. Rev Gauch Enferm. 2010 dez; 31(4): 701-07.

16. Gomes R. Descentralizando o atendimento ao usuário do sistema de saúde do município de Volta Redonda-RJ como forma de melhorar a resolutividade da atenção básica. Volta Redonda: Secretaria Municipal de Saúde; 2007.

17. Queiroz, MVO, Lucena NBF, Brasil EGM, Gomes ILV. Cuidado ao adolescente na atenção primária: discurso dos profissionais sobre 0 enfoque da integralidade. Rev RENE. 2011; 12 (n. esp): 1036-44.

\section{NOTAS}

${ }^{a} 0$ artigo refere-se ao recorte da dissertação: Processo de cuidar ao adolescente na atenção básica apresentada ao Curso de Mestrado Acadêmico em Cuidados Clínicos da Universidade Estadual do Ceará, defendida em 18/01/2011 